\title{
ASYMPTOTIC BEHAVIOUR OF MEASURE-VALUED CRITICAL BRANCHING PROCESSES
}

\author{
ALISON M. ETHERIDGE
}

(Communicated by Lawrence F. Gray)

\begin{abstract}
Measure-valued branching processes can be characterized in terms of the Laplace transform of their transition densities and this gives rise to a second order nonlinear p.d.e.- the evolution equation of the process. We write the solution to this evolution equation as a series, each of whose coefficients is expressed in terms of the linear semigroup corresponding to the spatial part of the measure-valued process. From this we obtain a simple proof that if the spatial part of the process is a recurrent (resp., transient) Markov process on a standard Borel space and the initial value of the process is an invariant measure of this spatial process, then the process has no (resp., has a unique) nontrivial limiting distribution.
\end{abstract}

\section{INTRODUCTION}

Measure-valued critical branching diffusion processes were first constructed by Watanabe in 1968 [14]. They arise as weak limits of branching diffusions when we change the scales of mass and lifetime in an appropriate way. The origin of such processes is as a model of the growth of a population of rare mutant genes in a large normal background. Feller in 1951 [10] observed that while a model of the size of a small population should be discrete, for large populations we may apply a continuous approximation, and this leads to a diffusion process. The measure-valued branching processes may be thought of as the extension of this idea of "diffusion approximation" to the space-time context. We may construct them on any standard Borel space on which we have defined a regular Markov process. In this paper we are concerned with the asymptotic behaviour of such processes.

All Markov processes considered below are assumed to be regular.

Remark. The processes introduced by Watanabe take their values among finite measures on $\mathbb{R}^{d}$. The construction of branching processes that take their values

Received by the editors April 8, 1991.

1991 Mathematics Subject Classification. Primary 60J80, 60J60, 60J57, 60F05; Secondary $35 \mathrm{C} 10,35 \mathrm{C} 20,35 \mathrm{~K} 55$.

Key words and phrases. Measure-valued diffusions, critical branching process, diffusion approximation, asymptotic distribution.

The author is a SERC Postdoctoral Research Fellow. Work largely completed while Sir Christopher Cox Junior Research Fellow, New College, Oxford. 
among finite measures on standard Borel spaces and whose spatial part is not necessarily a diffusion process is due to Dynkin [4]. Although one can proceed in a way entirely similar to the case of $\mathbb{R}^{d}$ (as outlined in $\S 2$ ), his construction has the advantage that it does not involve any passage to the limit.

The measure-valued processes constructed by Watanabe (and Dynkin [14]) all have finite initial mass, and from their Laplace transform characterization it is easy to see that the expected total mass tends to zero as time increases to infinity. However, as we describe in $\S 2$, we may use these finite mass processes to construct processes started from certain infinite measures (Dawson [2], Iscoe [12], Etheridge [8]). The question of asymptotic behaviour is then a more interesting (and a more difficult) one. If stable limiting distributions are going to exist for these processes, then one would expect each such limit to have some sort of "catchment area" and so we should choose their initial distribution with care. An obvious candidate for the initial value of the measure-valued process (which also turns out to simplify the calculations) is an invariant measure of the spatial part of the process. If the spatial part of the measure-valued process is Brownian motion or a symmetric stable process on $\mathbb{R}^{d}$, then Dawson [3] shows that if we take the initial value to be Lebesgue measure then existence or nonexistence of a nontrivial limiting distribution is equivalent to transience or recurrence of the spatial motion. Since we may regard the measure-valued branching processes as continuous analogues of critical cluster fields with independent transition and branching mechanisms (where the clusters are replaced by random measures), the corresponding result for such processes, Kallenberg [13], suggests that this 'duality' should extend to the general case. That transience of the spatial motion implies existence of a nontrivial limiting distribution is a result of Dynkin [5]. Our aim in this article is to show that under some mild conditions recurrence of the spatial motion implies "local extinction" of the measure-valued process.

Definition 1.1. We shall say that the measure-valued process $X(t)$ suffers local extinction if for every compact set $K \subset E$ and every $\varepsilon>0$

$$
\lim _{t \rightarrow \infty} \mathbb{P}\left[\left\langle\chi_{K}, X(t)\right\rangle>\varepsilon\right]=0
$$

where $\chi_{K}$ is the indicator function of the set $K$.

Recall that a function $l(t)$ on $(0, \infty)$ is said to be slowly varying at infinity if for every fixed $x, l(t x) / l(t) \rightarrow 1$ as $t \rightarrow \infty$.

We will denote by $C_{b}^{+}(E)$ the continuous, bounded, positive functions on $E$.

Our main result is the following.

Theorem 1.2. Let $E$ be a locally compact separable metric space with Borel field $\mathscr{B}_{E}$. Suppose that $X(t)$ is a measure-valued critical branching process whose spatial part is a recurrent (regular) Markov process on $E$ with transition semigroup $\left\{T_{t}\right\}_{t \geq 0}$ satisfying the following:

$\exists$ a function $h(t)$ on $(0, \infty)$, finite constants $C, \varepsilon, \varepsilon^{\prime}>0$ and an invariant measure $\mu$ of the spatial process such that for $\phi \in C_{b}^{+}(E)$,

(a)

$$
\frac{1}{h(t)} \int_{0}^{t} T_{s} \phi d s \rightarrow \int \phi d \mu \quad \text { as } t \rightarrow x
$$


(b)

$$
T_{t} \phi(x) \leq \frac{1}{2} C \cdot \frac{d h}{d t}(t) \int \phi d \mu \quad \text { uniformly in } x \text { as } t \rightarrow \infty ;
$$

(c) $\int_{G} T_{t} \phi d \mu>\varepsilon \phi d \mu$ as $t \rightarrow \infty$, where

$$
G=\left\{x: T_{t} \phi(x)>\varepsilon^{\prime} \frac{d h}{d t}(t) \int \phi d \mu\right\}
$$

(d) $h(t) \sim t^{\rho l}(t)$ as $t \rightarrow \infty$ for some slowly varying function $l(t)$ and some $0 \leq \rho \leq 1$.

Further, suppose that the variance of the branching part of $X(t)$ is a continuous function of $x \in E$ that is both uniformly bounded and uniformly strictly positive. Then the measure-valued process with initial value $X(0)=\mu$ suffers local extinction.

(Notice that $h(t) \rightarrow \infty$ as $t \rightarrow \infty$ ).

That Markov processes satisfying such conditions form a large class follows from, for example, Carlen, Kusuoka, and Stroock [1]. In particular, the spatial process could be any recurrent diffusion process on $\mathbb{R}^{d}$ generated by a uniformly strictly elliptic second-order differential operator with bounded continuous coefficients. Condition (c) seems to be the most natural way to ensure that

$$
\iint_{0}^{t} T_{s} \phi \cdot T_{s} \phi d s d \mu>\beta h(t)\left(\int \phi d \mu\right)^{2} \quad \text { as } t \rightarrow \infty
$$

for some $\beta>0$.

Dawson's approach in the special cases mentioned above trivially extends to yield an elementary proof of existence of nontrivial limiting distributions for measure-valued critical branching processes with transient spatial part (started from an invariant measure of this spatial part). However, his approach to the recurrent case rests heavily on precise knowledge of the transition densities of Brownian motion and the symmetric stable processes, so we have to adopt a different approach (although the use of a series solution to the evolution equation is inspired by his proof in the transient case).

In $\S 2$ we give a precise definition of measure-valued critical branching processes and a characterization of them via the Laplace transforms of their transition densities. This leads us to consider a second-order nonlinear p.d.e.- the evolution equation. In $\S 3$ we express the solution to this equation as an infinite sum, each of whose terms is expressed in terms of the linear semigroup associated with the spatial part of our process. In establishing the convergence of this series, we obtain upper bounds on the rate of growth of its coefficients, and condition (c) of Theorem (1.2) yields a lower bound on the rate of growth of the second-order term (when the spatial part of the process is recurrent). These estimates lead in $\S 4$ to a simple proof of our result.

Remark. If the spatial part of the process is positive recurrent, then using the same Tauberian methods it is possible to establish the exact asymptotic behaviour of the coefficients of the series solution (Etheridge $[8,9])$. Of course, in this case, the invariant measure has finite total mass so that it is easy to establish extinction of the measure-valued process, but we can construct processes with "immigration of mass" that do not die out and it is in this context in $[8,9]$ that we use the knowledge of this asymptotic behaviour. 
For easy reference we gather together in the Appendix some results on ordered binary rooted trees.

\section{DEFINITION OF THE PROCESSES}

We take $E$ to be a locally compact separable metric space with Borel field $\mathscr{B}_{E}$. Let $\mathcal{N}$ denote the purely atomic measures on $E$. Recall that a multiplicative Markov particle system is an $\mathscr{N}$-valued Markov process whose transition probabilities, $\left\{P_{t}\right\}_{t \geq 0}$, satisfy the so-called branching property

$$
P_{t}\left(\cdot, \nu_{1}+\nu_{2}\right)=P_{t}\left(\cdot, \nu_{1}\right) * P_{t}\left(\cdot, \nu_{2}\right) \quad \forall \nu_{1}, \nu_{2} \in \mathcal{N}, \forall t \geq 0
$$

(where $*$ denotes convolution); i.e., the distribution of the process with initial value $\nu_{1}+\nu_{2}$ is equal to that of the sum of two independent copies of the process with initial values $\nu_{1}, \nu_{2}$, respectively.

Definition 2.1. A branching Markov process, $\left(Z_{t}\right)_{t \geq 0}$, is a multiplicative particle system in which the particles independently undergo a random motion in $E$ and branching described as follows.

(a) Spatial motion. Until the time that it branches, each particle (independently of all other particles) performs a Markov process with generator $L$, say, in $E$.

(b) The branching rate. The probability that a particle located at $x \in E$ at time $t$ branches in the interval $[t, t+\delta t)$ is $V(x) \delta t+o(\delta t) . V(x)$ is called the branching rate.

(c) The branching mechanism. When a particle 'branches,' it dies and is replaced at the same location by a random number of offspring, governed by what we call the branching mechanism. We denote the generator of the branching mechanism by $\Phi(s, x)$. Thus, if $p_{k}(x)$ is the probability that a particle that dies at $x$ gives rise to $k$ offspring, then

$$
\Phi(s, x)=\sum_{k=0}^{\infty} p_{k}(x) s^{k}, \quad x \in E, s \in \mathbb{R} .
$$

Notice, that, by altering $V(x)$ if necessary, we may as well assume that $p_{1}(x)$ $\equiv 0$.

We shall call this the $(L, V \Phi)$-branching Markov process and refer to the Markov process generated by $L$ as its spatial part or base process. We write $\mathscr{D}(L)$ for the domain of the generator $L$ and $\left\{T_{t}\right\}_{t \geq 0}$ for the corresponding expectation semigroup.

Assumption. Throughout this article we shall assume that if the spatial part of the process is recurrent, then it satisfies the conditions of Theorem 1.2.

Suppose first that we are constructing the measure-valued process starting from a finite initial measure. We apply Feller's rescaling to these $\mathcal{N}$-valued processes. The idea is that the mass of each particle tends to zero as the number of particles tends to infinity, the total mass remaining constant. Specifically, let $Z^{n}(t)$ denote the $(L, n V \Phi)$-branching Markov process and let $X^{n}(t)=$ $\frac{1}{n} Z^{n}(t)$

Theorem 2.2. If $\left\{Z^{n}(0)\right\}_{n \geq 1}$ are chosen in such a way that the sequence $\left\{X^{n}(0)\right\}_{n \geq 1}$ of finite measures converges weakly as $n \rightarrow \infty$ to a limit $\mu$, say, 
then $\left\{X^{n}(\cdot)\right\}_{n \geq 1}$ converges weakly as $n \rightarrow \infty$ to a measure-valued process $X(\cdot)$, say, with $X(0)=\mu$. Furthermore, $X(\cdot)$ can be characterized as follows. For $\phi \in C_{b}^{+}(E) \cap \mathscr{D}(L)$

$$
\mathbb{E}_{\mu}[\exp (-\langle\phi, X(t)\rangle)]=\exp (-\langle u(t), \mu\rangle)
$$

where

$$
\begin{gathered}
\frac{\partial u}{\partial t}=L u-b u^{2} \\
u(0)=\phi \\
b(x)=\frac{1}{2} V(x)\left(\left.\frac{\partial^{2}}{\partial s^{2}} \Phi(s, x)\right|_{s=1}\right) .
\end{gathered}
$$

Remark. It would be more natural to write $u=u(t, x)$, but for conciseness we suppress the dependence on $x$ in our notation.

The reader is referred to Ethier and Kurtz [7, Chapter 9] for a "hi-tech" proof of this result, or to Etheridge [8] for a more direct attack. Dynkin [6] shows that the measure-valued processes constructed in this way are regular Markov processes, and with a little more work, it can be shown that if the spatial part of the approximating processes is a diffusion then so is the measurevalued limit. The measure-valued branching diffusion on $\mathbb{R}^{d}$ corresponding to Brownian motion and constant $b$ is known as the Dawson process.

We will call $b(x)$ the variance of the branching mechanism of $X(\cdot)$. Recall the assumption of Theorem 1.2 that $b$ is uniformly bounded both above and away from zero.

Equation (2.1) is called the evolution equation of the measure-valued branching process. We can extend the characterization to include more general $\phi$ by casting it into so-called "mild" form. Any solution of (2.1) solves the integral equation

$$
u(t)=T_{t} u(0)-\int_{0}^{t} T_{t-s}\left(b \cdot u(s)^{2}\right) d s .
$$

A solution of $\left(2.1^{\prime}\right)$ is called a mild solution of $(2.1)$. It can be shown that for each $\phi \in C_{b}^{+}(E),\left(2.1^{\prime}\right)$ has a unique positive solution with $u(0)=\phi$, and that if in addition $\phi \in \mathscr{D}(L)$ then this solution also solves (2.1). (This is proved in Iscoe [12] or can be deduced with a little more work from the representation of the solution to $\left(2.1^{\prime}\right)$ as a series given in $\S 3$.) In fact, we can even solve the integral equation with initial values of the form $\phi=\chi_{K}$, the indicator function of a compact set.

As we remarked in $\S 1$, we are really interested in such processes started from infinite initial measures. In order to achieve a sensible definition of such a process, we should like to avoid the possibility that an infinite amount of mass might "cascade in" from infinity into a compact set in a finite time, and for this reason, we cannot take arbitrary initial values. A natural way to try to construct the measure-valued process starting from an infinite initial measure $\mu$, would be to decompose $\mu$ into a countable number of finite measures, $\left\{\mu_{i}\right\}_{i \in \mathbb{N}}$ say, and exploit the multiplicativity of the process. From our Laplace transform characterization of the process started from $\mu_{i}$, the measure-valued process 
started from $\mu$ (if it exists) will satisfy

$$
\begin{aligned}
\mathbb{E}_{\mu}[\exp (-\langle\phi, X(t)\rangle)] & =\prod_{i=1}^{\infty} \mathbb{E}_{\mu_{i}}[\exp (-\langle\phi, X(t)\rangle)] \\
& =\prod_{i=1}^{\infty} \exp \left(-\left\langle u(t), \mu_{i}\right\rangle\right)=\exp (-\langle u(t), \mu\rangle)
\end{aligned}
$$

where $u(t)$ satisfies the usual evolution equation with $u(0)=\phi$. Thus to avoid the problem of "cascading mass" we should choose $\mu$ in such a way that

$$
\int u(t) d \mu<\infty \quad \forall t \geq 0, \forall \phi \in C_{K}(E) .
$$

$\left(C_{K}(E)\right.$ is the space of continuous functions of compact support on $E$.) Unfortunately, $u(t)$ is difficult to write down explicitly. However, we do have an obvious upper bound: $u(t) \leq T_{t} u(0) \forall t \geq 0$. It can be shown (see [8]) that if we choose an initial measure $\mu$ for which

$$
\int T_{t} \phi d \mu<\infty \quad \forall t \geq 0, \forall \phi \in C_{K}(E),
$$

then we can construct a unique measure-valued process by decomposing $\mu$ in this way (the difficulty is to check that the path properties of the process are preserved under this type of summation. Iscoe [12] uses a different approach to prove a similar result in the case where the spatial part of the process is a symmetric stable process.) In particular, if $\mu$ is an invariant measure of the spatial part of the process, then we can construct the process started from $\mu$. It is the measure-valued branching process with this initial condition that we study here.

\section{A SERIES SOLUTION OF THE EVOLUTION EQUATION}

In order to establish the asymptotic behaviour of the measure-valued processes, we should like to have some expression for the solution to the mild form of the evolution equation, at least for $u(0)=\lambda \phi$ where $\lambda$ is some strictly positive constant and $\phi$ has compact support in $E$.

We proceed formally. Define a multiplication operator $\mathscr{S}$ by

$$
\mathscr{S}(f \circ g)(t)=-\int_{0}^{t} T_{t-s}(b \cdot f(s) \cdot g(s)) d s .
$$

Equation $\left(2.1^{\prime}\right)$ becomes

$$
u(t)=T_{t} u(0)+\mathscr{S}(u \circ u)(t) .
$$

It is easy to prove that $\mathscr{S}$ is distributive, but not, in general, associative. We try to solve (3.1) iteratively. Define

$$
\begin{aligned}
u_{0}(t) & =T_{t} u(0), \\
u_{r+1}(t) & =T_{t} u(0)+\mathscr{S}\left(u_{r} \circ u_{r}\right)(t) .
\end{aligned}
$$

If the series $\left\{u_{r}\right\}_{r \geq 0}$ converges to a limit $u$, then $u$ will solve (3.1). We expand 
the first few members of the sequence

$$
\begin{aligned}
u_{1}= & u_{0}+\mathscr{S}\left(u_{0} \circ u_{0}\right) \\
u_{2}= & u_{0}+\mathscr{S}\left(\left(u_{0}+\mathscr{S}\left(u_{0} \circ u_{0}\right)\right) \circ\left(u_{0}+\mathscr{S}\left(u_{0} \circ u_{0}\right)\right)\right) \\
= & u_{0}+\mathscr{S}\left(u_{0} \circ u_{0}\right)+\mathscr{S}\left(u_{0} \circ \mathscr{S}\left(u_{0} \circ u_{0}\right)\right)+\mathscr{S}\left(\mathscr{S}\left(u_{0} \circ u_{0}\right) \circ u_{0}\right) \\
& +\mathscr{S}\left(\mathscr{S}\left(u_{0} \circ u_{0}\right) \circ \mathscr{S}\left(u_{0} \circ u_{0}\right)\right) .
\end{aligned}
$$

Continuing in this way, it is clear that there is a one-to-one correspondence between terms of the expansion of this type of $u_{r}$ and the set $\mathscr{T}_{r}$ of ordered binary rooted trees of order $\leq 2^{r}$. (The reader is referred to the appendix for definitions and results related to such trees.) Indeed we may write

$$
u_{r}(t)=\sum_{\tau \in \mathscr{T}_{r}} U_{t}^{\tau}(u(0))
$$

where $\left\{U_{t}^{\tau}(u(0))\right\}$ are defined inductively as follows. If $\tau_{e}$ is the tree of order 1 and $\tau_{1} \circ \tau_{2}$ denotes the tree formed by linking the roots of trees $\tau_{1}$ and $\tau_{2}$ to a common root (thus forming a tree of order $\left.\left|\tau_{1}\right|+\left|\tau_{2}\right|\right)$ then $U_{t}^{\tau_{e}}(u(0))=T_{t} u(0)$,

$$
U_{t}^{\tau_{1} \circ \tau_{2}}(u(0))=\mathscr{S}\left(U^{\tau_{1}}(u(0)) \circ U^{\tau_{2}}(u(0))\right)(t) .
$$

Formally then $u(t)=\sum_{\tau \in \mathscr{T}} U_{t}^{\tau}(u(0))$ is a solution to (3.1) (the sum being over all ordered binary rooted trees). Suppose that $u(0)=\lambda \phi$. This becomes

$$
u(t)=\sum_{\tau \in \mathscr{T}} U_{t}^{\tau}(\lambda \phi)=\sum_{\tau \in \mathscr{T}} \lambda^{|\tau|} U_{t}^{\tau}(\phi) .
$$

Remark. Sums of this type are often called Wild sums. Wild [15] used this construction to produce a series solution of Boltzmann's equation.

We investigate the convergence of this power series for semigroups $\left\{T_{t}\right\}_{t \geq 0}$ satisfying the conditions of Theorem 1.2. Let $T(t, \phi)=C h(t) \int \phi d \mu$ and write $\tau_{s}=\tau_{e} \circ \tau_{e}$. Then it is easy to see that (under assumption (b) of Theorem 1.2)

$$
-U_{t}^{\tau_{s}}(\lambda \phi)(x) \leq \bar{b} \cdot \lambda^{2} \cdot T(t, \phi) \cdot\left(T_{t} \phi\right)(x) \quad \text { where } \bar{b}=\sup _{x} b(x) .
$$

Proceeding inductively, we have

$$
\left|U_{t}^{\tau}(\lambda \phi)(x)\right| \leq \lambda^{|\tau|}(\bar{b} T(t, \phi))^{|\tau|-1}\left(T_{t} \phi\right)(x) .
$$

Now, from Theorem (A.1), if $C(n)$ denotes the number of ordered binary rooted trees of order $n$, then the series

$$
\sum_{n=1}^{\infty} C(n) \lambda^{n}(\bar{b} T(t, \phi))^{n-1}
$$

is convergent for $\lambda \bar{b} T(t, \phi)<\frac{1}{4}$. Thus, for $\lambda<1 / 8 \bar{b} T(t, \phi)$, say, the sum $\sum_{\tau \in \mathscr{G}} U_{t}^{\tau}(\lambda \phi)(x)$ is absolutely and uniformly (in both $\lambda$ and $x$ ) convergent to a uniformly bounded limit.

We also require a lower bound on $-\int U_{t}^{\tau_{s}}(\lambda \phi) d \mu$ as $t \rightarrow \infty$. Using assumption (c) of Theorem (1.2), Fubini's Theorem, and the fact that $\mu$ is an invariant 
measure of the spatial motion,

$$
\begin{aligned}
\frac{d}{d t}( & \left.-\int U_{t}^{\tau_{s}}(\lambda \phi) d \mu\right)=\lambda^{2} \frac{d}{d t}\left(\iint_{0}^{t} T_{t-s}\left(T_{s} \phi \cdot T_{s} \phi\right) d s d \mu\right) \\
& =\lambda^{2} \frac{d}{d t}\left(\iint_{0}^{t}\left(T_{s} \phi \cdot T_{s} \phi\right) d s d \mu\right)=\lambda^{2} \int T_{s} \phi \cdot T_{s} \phi d \mu \\
& \geq \lambda^{2} \varepsilon^{\prime} \frac{d h}{d t} \int \phi d \mu\left(\int_{G} T_{t} \phi d \mu\right) \geq \lambda^{2} \varepsilon \varepsilon^{\prime} \frac{d h}{d t}\left(\int \phi d \mu\right)^{2} \quad \text { as } t \rightarrow \infty
\end{aligned}
$$

Since $h(t) \rightarrow \infty$ as $t \rightarrow \infty$, this implies

$$
-\int U_{t}^{\tau_{s}}(\lambda \phi) d \mu \geq \lambda^{2} \beta h(t)\left(\int \phi d \mu\right)^{2} \quad \text { as } t \rightarrow \infty
$$

where $\beta=\frac{1}{2} \varepsilon \varepsilon^{\prime}$.

Remark. Notice that repeatedly differentiating $\mathbb{E}_{x(0)}[\exp (-\langle\lambda \phi, X(t)\rangle)]$ with respect to $\lambda$ and evaluating at $\lambda=0$ yields expressions for the moments of the measure-valued process in terms of the coefficients of this power series. Thus the estimates above actually amount to moment estimates for the measure-valued process.

\section{Proof of Theorem 1.2}

Before fulfilling our aim and proving Theorem 1.2, we indicate how the binary tree technique leads us to a straightforward proof of the existence of nontrivial limiting distributions for measure-valued critical branching processes with transient spatial part. It is a simple extension of the corresponding proof of Theorem (4.1) in [3]. If the spatial part of the process is assumed transient, then we can mimic our approach in $\S 3$, except that $T(t, \phi)$ is now replaced by a finite constant independent of $t$, so that for sufficiently small $\lambda$ our series solution to the evolution equation with initial condition $u(0)=\lambda \phi$ is valid for all $t$. Using the fact that $\mu$ is an invariant measure of the spatial process and interchanging the order of summation and integration (justified by uniform convergence), it is an easy matter to see that $\int u(t) d \mu$ converges to a bounded limit. Moreover this limit is nontrivial for at least some $\lambda$. The reader is referred to [3] for further details (although Dawson uses the characteristic functional in place of the Laplace transform).

Consider now the case where the spatial process corresponding to $T_{t}$ is null recurrent. We will use the following well-known result.

Theorem 4.1 (see [11, Vol. II, Chapter XIII, Theorem 2]). For $n=1,2, \ldots$ let $F_{n}$ be a probability distribution with Laplace transform $\phi_{n}$.

If $F_{n} \rightarrow F$, where $F$ is a possibly defective distribution with Laplace transform $\phi$, then $\phi_{n}(\lambda) \rightarrow \phi(\lambda)$ for $\lambda>0$.

Our proof of theorem 1.2 also requires the following

Lemma 4.2. Suppose that $0<\beta, C<\infty$. Then we can find $\alpha \in(0,1)$ with

$$
\frac{1}{\alpha}(\sqrt{1+\alpha}-\sqrt{1-\alpha})<1+\frac{\alpha \beta}{16 C} .
$$


Proof. Equivalently we seek $\alpha$ for which

$$
2<\left(1+\frac{\alpha \beta}{16 C}\right)(\sqrt{1+\alpha}+\sqrt{1-\alpha}) .
$$

Notice then that the function on the right-hand side is smooth on $[0,1)$ and is equal to 2 at 0 , with strictly positive first derivative at 0 . The result follows.

Proof of Theorem 1.2. Most of the work was done in $\S 3$. We argue by contradiction. Suppose that the process does converge to a nontrivial limit. Then, by theorem 4.1, we must have that for any $\phi \in C_{b}(E), \int u(t) d \mu$ converges to a finite limit as $t \rightarrow \infty$ and, furthermore, this limit is nontrivial for at least some $\phi_{0}$. Let us choose such a $\phi_{0}$, and suppose that $T$ is chosen sufficiently large so that

$$
\left(1-\frac{\alpha \beta}{16 C}\right) \int u(T) d \mu<\lim _{t \rightarrow \infty} \int u(t) d \mu,
$$

where $\beta$ is chosen (as in (3.4)) in such a way that $\left(\forall \phi \in C_{b}^{+}(E)\right)$

$$
-\int U_{t}^{\tau_{s}} \phi d \mu \geq \beta h(t)\left(\int \phi d \mu\right)^{2} \quad \text { as } t \rightarrow \infty
$$

and $\alpha$ is chosen as in Lemma 4.2. We show that there is a finite time $\tilde{T}$ at which

$$
\int u(T+\widetilde{T}) d \mu<\left(1-\frac{\alpha \beta}{16 C}\right) \int u(T) d \mu
$$

and, since $\int u(t) d \mu$ is monotone, thereby arrive at a contradiction.

Consider once again the series solution to the evolution equation. We write $u_{\lambda}(t)$ for the solution to the evolution equation with $u_{\lambda}(0)=\lambda u(0)$. Since the spatial process is recurrent, we have seen that we cannot find $\lambda>0$ for which the power series expansion for $u_{\lambda}(t)$ converges for all $t$. However, for fixed $t>0$, we do have convergence for $\lambda=\lambda(t)<(4 T(t, \phi))^{-1}=\left(4 C h(t) \int \phi d \mu\right)^{-1}$. We set $\phi=u(T)$ in this series and, for convenience of notation, consider our time scale to have 'restarted' with $T=0$. We will exploit the sublinearity of the evolution equation. Observe then that for $\lambda \leq 1, u_{1}(t) \leq(1 / \lambda) u_{\lambda}(t)$. We set

$$
\lambda(t)=\alpha / 4 C h(t) \int \phi d \mu, \quad 0 \leq \alpha<1-\varepsilon
$$

(where $\varepsilon$ is a small positive constant). From $\S 3$, for this value of $\lambda$ we may express $\int u_{\lambda(t)}(t) d \mu / \lambda(t)$ as a series. (The role of $\varepsilon$ is to ensure that we are strictly within the radius of convergence of the power series for $u_{\lambda(t)}(t)$, so that we may interchange the order of integration and summation.) Consider the first two terms

$$
\begin{gathered}
\frac{1}{\lambda(t)} \int U_{t}^{\tau_{e}}(\lambda(t) \phi) d \mu=\int \phi d \mu, \\
\frac{1}{\lambda(t)} \int U_{t}^{\tau_{s}}(\lambda(t) \phi) d \mu \leq-\lambda(t) \beta h(t)\left(\int \phi d \mu\right)^{2}=-\frac{\alpha \beta}{4 C} \int \phi d \mu \quad \text { as } t \rightarrow \infty .
\end{gathered}
$$

Thus, at least for sufficiently large $T$, the contribution to the sum from the first two terms is bounded above by

$$
\left(1-\frac{\alpha \beta}{8 C}\right) \int \phi d \mu \text {. }
$$


Consider then the sum of the remaining positive terms of the series. These come from trees of odd order and from $\S 3$ we have that

$$
\left|\int U_{t}^{\tau}(\lambda(t) \phi) d \mu\right| \leq(C h(t))^{|\tau|-1}\left(\lambda(t) \int \phi d \mu\right)^{|\tau|}=\frac{1}{C h(t)}\left(\frac{\alpha}{4}\right)^{|\tau|} \quad \text { as } t \rightarrow \infty .
$$

From the appendix, if $C(n)$ denotes the number of binary rooted trees of order $n$, then

$$
\sum_{n=1}^{\infty} C(n) \theta^{n}=\frac{1}{2}(1-\sqrt{1-4 \theta}) .
$$

Thus the sum of the positive terms of order greater than one in our expansion of $\int u_{\lambda(t)} d \mu / \lambda(t)$ is bounded above (for large enough $t$ ) by

$$
\left(\frac{1}{4}(\sqrt{1+\alpha}-\sqrt{1-\alpha})\right) \int \phi d \mu \cdot \frac{4}{\alpha}-\int \phi d \mu .
$$

But by our choice of $\alpha$, this is less than

$$
\frac{\alpha \beta}{16 C} \int \phi d \mu,
$$

and this leads us to the required contradiction.

Remark. The proof above uses the crudest of estimates and so almost certainly disguises the rather rapid rate of decay of the process at least in a compact set.

\section{APPENDIX. ORDERED BINARY ROOTED TREES}

We may construct the set of ordered binary rooted trees, $\mathscr{T}$, inductively. We let $\tau_{\varepsilon}$ denote the tree consisting of a single vertex. Given two trees $\tau_{1}$ and $\tau_{2}, \tau_{1} \circ \tau_{2}$ is the tree obtained by connecting the roots of $\tau_{1}$ and $\tau_{2}$ to a single vertex. For our purposes in $\S 3$ we must consider ordered trees, i.e., the two trees $\tau_{1} \circ \tau_{2}$ and $\tau_{2} \circ \tau_{1}$ are deemed to be different if $\tau_{1} \neq \tau_{2}$. The order $|\tau|$ of $\tau \in \mathscr{T}$ is defined to be the number of vertices having at most one neighbour. Thus $\left|\tau_{1} \circ \tau_{2}\right|=\left|\tau_{1}\right|+\left|\tau_{2}\right|$. The result that we require is

Theorem A.1. Let $C(n)$ denote the number of ordered binary rooted trees of order $n$. Then the power series

$$
\sum_{n=1}^{\infty} C(n) \theta^{n}
$$

has radius of convergence $\frac{1}{4}$.

Proof. From the construction described above, given any such tree of order $n \geq 2$ we can decompose it into two trees of lower order (by removing the root). It is easy to see that we have the recurrence relation

$$
C(n)=\sum_{j=1}^{n-1} C(j) C(n-j) .
$$


Consider the complex function $f(z)=\sum_{n=1}^{\infty} C(n) z^{n}$. Suppose that this series converges.

$$
\begin{aligned}
(f(z))^{2} & =\left(\sum_{n=1}^{\infty} C(n) z^{n}\right)^{2}=\sum_{k=1}^{\infty} \sum_{i=1}^{k} C(i) C(k+1-i) z^{k+1} \\
& =\sum_{n=2}^{\infty} \sum_{i=1}^{n} C(i) C(n-i) z^{n}=\sum_{n=2}^{\infty} C(n) z^{n}=f(z)-z .
\end{aligned}
$$

Thus if the series converges we have $(f(z))^{2}+z=f(z)$. Solving this quadratic and choosing $f(0)=0$, we obtain $f(z)=\frac{1}{2}(1-\sqrt{1-4 z})$, where $f(z)$ is holomorphic on the disc of radius $\frac{1}{4}$ and its power series is (A.1), which implies the result.

\section{ACKNOWLEDGMENT}

The author would like to thank the referee for his care and patience in reading earlier versions of this paper.

\section{REFERENCES}

1. E. A. Carlen, S. Kusuoka, and D. W. Stroock, Upper bounds for symmetric Markov transition functions, Ann. Inst. H. Poincaré 23 (1987), 245-287.

2. D. A. Dawson, Stochastic evolution equations and related measure processes, J. Multivariate Anal. 5 (1975), 1-52.

3. _ The critical measure diffusion process, Z. Warsch. Verw. Gebiete 40 (1977), 125-145.

4. E. B. Dynkin, Superprocesses and their linear additive functionals, Trans. Amer. Math. Soc. 314 (1989), 255-282.

5. __ Three classes of infinite dimensional diffusions, J. Funct. Anal. 86 (1989), 75-110.

6. __ Regular transition functions and regular superprocesses, Trans. Amer. Math. Soc. 316 (1989), 623-634.

7. S. N. Ethier and T. G. Kurtz, Markov processes: characterization and convergence, Wiley, New York, 1986.

8. A. M. Etheridge, Asymptotic behaviour of some measure-valued diffusions, Oxford Ph.D. thesis, 1989.

9. __ Measure-valued critical branching diffusion processes with immigration, unpublished manuscript.

10. W. Feller, Diffusion processes in genetics, Proc. 2nd Berkeley Sympos. Math. Statist. Prob., Univ. of California Press, Berkeley, CA, 1951, pp. 227-246.

11. __ An introduction to probability theory and its applications vol. II, Wiley, New York, 1966.

12. I. Iscoe, $A$ weighted occupation time for a class of measure-valued branching processes, Probab. Theory Rel. Fields 71 (1986), 85-116.

13. O. Kallenberg, Stability of critical cluster fields, Math. Nachr. 77 (1975), 7-43.

14. S. Watanabe, $A$ limit theorem of branching processes and continuous state branching processes, J. Math. Kyoto Univ. 8 (1968), 141-167.

15. E. Wild, On Boltzmann's equation in the kinetic theory of gases, Math. Proc. Cambridge Philos. Soc. 47 (1951), 602-609.

The Statistical laboratory, 16, Mill Lane, Cambridge, United Kingdom

Current address: Department of Mathematics and Statistics, University of Edinburgh, JCMB,

The King's Building, Mayfield Rd., Edinburgh, EH9 3JZ, United Kingdom

E-mail address: alison@castle.edinburgh.ac.uk or alison@maths.ed.ac.uk 